\title{
miR-150, p53 protein and relevant miRNAs consist of a regulatory network in NSCLC tumorigenesis
}

\author{
DE-TAO WANG $^{1 *}$, ZHONG-LIANG MA $^{1 *}$, YAN-LI LI ${ }^{1}$, YUE-QING WANG $^{1}$, BO-TAO ZHAO $^{1}$, \\ JIA-LI WEI $^{1}$, XIANG QIU ${ }^{1}$, XIN-TAI ZHAO ${ }^{3}$ and YOU-XIN JIN ${ }^{1,2}$ \\ ${ }^{1}$ School of Life Sciences, Shanghai University, Shanghai 200444; ${ }^{2}$ State Key Laboratory of Molecular Biology, \\ Institute of Biochemistry and Cell Biology, Institutes for Biological Sciences, Chinese Academy of Sciences, \\ Shanghai 200031; ${ }^{3}$ Shanghai Shines Pharmaceuticals Co., Ltd., Shanghai 200032, P.R. China
}

Received February 22, 2013; Accepted April 3, 2013

DOI: $10.3892 /$ or.2013.2453

\begin{abstract}
RNAs (miRNAs) are a class of non-coding small RNAs that act as negative regulators of gene expression by binding to the 3'-untranslated region (3'-UTR) of target mRNAs. Tumor protein p53, a transcriptional factor, plays an important role in the progression of tumorigenesis. miR-150 was the only miRNA predicted to target 3'-UTR of $\mathrm{p} 53$ by TargetScan. In order to investigate the function of miR-150, p53 and relevant miRNAs in non-small cell lung cancer (NSCLC), we constructed two expression vectors of p53 (pcDNA3.1-p53 and pcDNA3.1-p53-3'-UTR) and two report vectors (pGL3-p53-3'-UTR and pGL3-p53-3'-mUTR). The activity of luciferase transfected with miR-150 mimics was lower by $30 \%$ when compared to that of the miRNAnegative control (miRNA-NC). Moreover, the p53 protein was downregulated by at least $50 \%$ when miR-150 mimics were cotransfected with pcDNA3.1-p53-3'-UTR when compared to miRNA-NC. We also determined the expression of miR-150 and p53 in NSCLC patient tissue samples. The expression of miR-150 in T2 stage tissue samples was higher than that in T1 stage tissue samples. The corresponding target gene p53 was correlated with miR-150 expression. In the present study, we further analyzed the cell cycle distribution. The cells transfected with pcDNA3.1-p53 were significantly arrested in the G1 phase when compared to the control cells. When miR-150 mimics were cotransfected with pcDNA3.1-p53-3'-UTR, the percentage of cells in the G1 phase was significantly lower by $4 \%$ when compared to miRNA-NC. To identify miRNAs that are regulated by the $\mathrm{p} 53$ protein, $\mathrm{qRT}$-PCR was performed after pcDNA3.1-p53 transfection. miR-34a, miR-184, miR-181a and
\end{abstract}

Correspondence to: Dr Zhong-Liang Ma or Dr You-Xin Jin, School of Life Sciences, Shanghai University, 99 Shangda Road, Shanghai 200444, P.R. China

E-mail: zlma@shu.edu.cn

E-mail: yxjin@sibs.ac.cn

"Contributed equally

Key words: miR-150, p53 protein, miRNA, NSCLC
miR-148 were upregulated significantly. However, there was no distinct difference in the expression of miR-10a, miR-182 and $\mathrm{miR}-34 \mathrm{c}$. Our results showed that miR-150 targets the 3'-UTR of p53, and p53 protein promotes the expression of miRNAs which affect cell cycle progression. These findings suggest that miR-150, p53 protein and relevant miRNAs are members of a regulatory network in NSCLC tumorigenesis.

\section{Introduction}

microRNAs (miRNA) are small non-coding RNA molecules that inhibit gene expression at the transcriptional and posttranscriptional level by binding to the 3'-untranslated region (3'-UTR) of target mRNAs (1-4). miRNAs can bind to partially complementary recognition sequences of mRNA, subsequently causing mRNA degradation or translation inhibition, thus effectively silencing their target genes (5-7). Bioinformatic analysis of known miRNAs suggests that the majority of mRNAs can be targeted by miRNAs and that a single miRNA can regulate several hundred genes $(8,9)$. miRNAs have been reported to participate in many important cellular processes, such as apoptosis, cell differentiation and proliferation, tumor suppression, development and metabolism (3,7-14). In recent years, more and more miRNAs have been detected by microarray analysis or other advanced technologies. At the same time, more protein factors have been confirmed to affect the expression of miRNAs, such as p53 $(12,15,16)$. Thus, in order to elucidate the molecular mechanisms associated with nonsmall cell lung cancer (NSCLC) cell cycle arrest, identification of the regulatory network of miRNAs and proteins is critical.

To identify miRNAs which are differentially expressed in NSCLC and corresponding non-tumor lung tissues, miRNA solexa analysis was performed. Seven miRNAs were chosen for further study. All of the candidate miRNAs which have been verified in our laboratory play an important role in NSCLC cell cycle arrest. Potential target genes of 7 miRNAs were predicted by TargetScan (Table I). All proteins can regulate the cell cycle. To further identify the miRNAs that may regulate the expression of $\mathrm{p} 53$ or be regulated by $\mathrm{p} 53$ protein, we performed a prediction using software and constructed two expression vectors of p53 (with or without 3'-UTR). Only miR-150 was predicted to bind to the 3'-UTR of p53 by TargetScan software. The p53 expression 
vector contained the coding sequence (cd) only (pcDNA3.1-p53) and cds with 3'-UTR containing the binding sequences of miR-150 (pcDNA3.1-p53-3'-UTR) were constructed, respectively. Our results showed that miR-150 targets the 3'-UTR of p53 and reduces G1 phase arrest in the H1299 cell line triggered by p53. miR-34a, miR-184, miR-181a and miR-148 were significantly upregulated in the H1299 cells transfected by pcDNA3.1-p53. Moreover, the expression of miR-34a and miR-184 was consistent with p53 in the NSCLC cell lines, including SPCA-1, H1299, A549 and HCC827. These findings suggest that miR-150, p53 protein and relevant miRNAs may be members of a regulatory network in NSCLC tumorigenesis.

\section{Materials and methods}

Cell culture. Human cell lines (SPCA-1, A549, HCC827, 95-D, HEK293T and BEAS-2B) were obtained from the Cell Bank of the China Academy of Sciences (Shanghai, China). H1299 was from the American Type Culture Collection (ATCC, Manassas, VA, USA). SPCA-1, A549, HCC827 and H1299 cell lines were derived from an NSCLC cell line, while 95-D is a small-cell lung cancer cell line with high metastatic potential. Human bronchial epithelial (BEAS-2B) cells were cultured in LHC-9 medium. A549 cells were cultured in F12K medium (Gibco, Gaithersburg, MD, USA). All the other lung cancer cells were cultured in RPMI-1640 medium (Gibco). Human embryonic kidney cells (HEK293T) were cultivated in Dulbecco's modified Eagle's medium (DMEM, Gibco). All the media were supplemented with $10 \%$ fetal bovine serum (FBS; HyClone Laboratories, Logan, UT, USA), $100 \mathrm{U} / \mathrm{ml}$ penicillin and $100 \mu \mathrm{g} /$ $\mathrm{ml}$ streptomycin. Cells were cultured at $37^{\circ} \mathrm{C}$ in $5 \% \mathrm{CO}_{2}$.

Clinical cancer samples. Human lung cancer samples were obtained from the Department of Oncology, Shanghai Chest Hospital affiliated to Shanghai Jiao Tong University, Shanghai, China, under ethical assessment.

Construction of recombinant expression vectors. The 3'-UTR of the tumor protein TP53 (p53) gene containing the miR-150 binding site (665 bp) was subcloned downstream of the firefly luciferase reporter gene in the pGL3 vector (Promega, Madison, WI, USA) and designated as pGL3-p53-3'-UTR. The plasmid pGL3-p53-3'-mUTR which contained the mutated binding site of miRNA-150 in the 3'-UTR was also constructed. The cds of p53 with or without the miR-150 binding sequence were cloned into the pcDNA3.1 (-) plasmid and named pcDNA3.1-p53 and pcDNA3.1-p53-3'-UTR, respectively. The primer sequences used in this study are shown in Table II.

Luciferase assay. For reporter assays, HEK293T cells cultured in 24-well plates were transiently cotransfected with $400 \mathrm{ng}$ luciferase vector pGL3-p53-3'-UTR or pGL3-p53-3'-mUTR and either miR-150 mimics or miRNA negative control (miRNA-NC). To determine the transfection efficiency, 20 ng pRL-SV40 (Promega) was cotransfected as the control. Reporter assays were performed at $36 \mathrm{~h}$ post-transfection using the Dual-luciferase assay system (Promega).

Quantitative real-time PCR ( $q R T-P C R)$ analysis of miRNAs and target genes. Total RNA was extracted from the cell
Table I. Potential target genes of 7 miRNAs predicted by TargetScan.

\begin{tabular}{|c|c|c|}
\hline miRNA & Target gene(s) & Published in TargetScan \\
\hline \multirow[t]{2}{*}{$\operatorname{miR}-34 a$} & CCNE2 & 2005, 2007, 2009 \\
\hline & CDKN1C & 2009 \\
\hline \multicolumn{3}{|l|}{ miR-184 } \\
\hline \multirow[t]{5}{*}{ miR-181a } & CAPRIN1 & 2005, 2007, 2009 \\
\hline & $\mathrm{CCNJ} / \mathrm{K}$ & 2009 \\
\hline & CCNT2 & 2005, 2007, 2009 \\
\hline & CCNG1 & \\
\hline & CDK8 & 2009 \\
\hline \multirow[t]{2}{*}{$\operatorname{miR}-148$} & CDK5R1 & $2005,2007,2009$ \\
\hline & CDK6/8/13/19 & $2003,2005,2007,2009$ \\
\hline \multirow[t]{2}{*}{ miR-10a } & CNNM4 & 2005,2007 \\
\hline & CDK6 & 2009 \\
\hline \multirow[t]{8}{*}{ miR-182 } & $\mathrm{CCNJ} / \mathrm{Y}$ & 2005, 2007, 2009 \\
\hline & GSPT1 & \\
\hline & CNNM2/3 & 2007, 2009 \\
\hline & CNGA3 & \\
\hline & $\mathrm{CCND} 2 / 3$ & \\
\hline & CDKN1C & \\
\hline & CDK6 & 2009 \\
\hline & CCNE2 & $2005,2007,2009$ \\
\hline \multirow[t]{2}{*}{ miR-34c } & CCNJL & 2009 \\
\hline & CCND1 & $2005,2007,2009$ \\
\hline
\end{tabular}

cultures using TRIzol reagent (Bio Basic Inc., Toronto, Canada) according to the manufacturer's instructions. Reverse transcription was performed using the M-MLV Reverse Transcriptase cDNA Synthesis kit (Takara, Dalian, China). A cDNA library of miRNAs was synthesized by the QuantiMir cDNA kit (Takara). U6 snRNA and the housekeeping gene 18S RNA were used as the endogenous control for miRNA and mRNA, respectively. The target genes and controls were treated under the same condition and analyzed by qRT-PCR using SYBR Premix Ex Taq ${ }^{\mathrm{TM}}$ (Takara) according to the manufacturer's protocol.

Western blot analysis. Protein for western blot analysis was precipitated according to the standard protocol (17-20). Equal amounts of protein samples were subjected to SDS-polyacrylamide gel electrophoresis (SDS-PAGE) and then transferred to a PVDF membrane. The membrane was soaked in Trisbuffered saline (TBS)-Tween buffer containing 5\% low-fat milk for 60 min with gentle shaking and then incubated with a specific antibody overnight followed by washing and incubating with a secondary antibody and the final chemiluminescence ECL (Thermo Scientific, Rockford, IL, USA) detection of the bands. Protein bands were quantitated by densitometric analysis using Image Lab analysis software and expressed as the fold of the control after being normalized to GAPDH. The primary antibodies used were rabbit anti-p53 $(1: 1,000)$ and mouse anti-GAPDH $(1: 1,000)$. The secondary 
Table II. Primer sequences used in this study.

Plasmid

Primer sequences

Restriction

enzyme

\begin{tabular}{llc}
\hline pGL3-p53-3'-UTR & Forward: CTAGTCTAGATCAGTCTACCTCCCGCCATAA & XbaI \\
& Reverse: CCGGAATTCTGACAACTCCCTCTACCTAACCAG & $E c o$ R1 \\
pGL3-p53-3'-mUTR & Forward: CTGTGAGGGATGTCTAGCATATGTAAGAAATGTTCTTGCAGTTAAGGG & \\
& Reverse: TTTCTTACATATGCTAGACATCCCTCACAGTAAAAACCTTAAAATCTAAGC & XhoI \\
pcDNA3.1-p53 & Forward: CCGCTCGAGATGGAGGAGCCGCAGTCAGA & $E c o$ 1 \\
& Reverse: CCGGAATTCCAAAACCCAAAATGGCAGGG & XhoI \\
pcDNA3.1-p53-3'-UTR & Forward: CCGCTCGAGATGGAGGAGCCGCAGTCAGA & $E c o$ R1 \\
& Reverse: CCGGAATTCCCCTACCTAGAATGTGGCTGATTG &
\end{tabular}

antibodies were rabbit anti-mouse $(1: 10,000)$ and mouse antirabbit $(1: 10,000)$. All antibodies were purchased from Cell Signaling Technology.

Cell cycle analysis. Cells were fixed in $70 \%$ ethanol for $12 \mathrm{~h}$ at $4^{\circ} \mathrm{C}$. After washing with phosphate-buffered solution (PBS), cells were treated with RNase A $(50 \mu \mathrm{g} / \mathrm{ml})$ and stained with propidium iodide (PI; $25 \mu \mathrm{g} / \mathrm{ml}$ ) for $30 \mathrm{~min}$ at $37^{\circ} \mathrm{C}$. Samples were analyzed using MoFlo XDP flow cytometer (Beckman Coulter, Inc., Brea, CA, USA) and the distribution of cell cycle phases was determined using FlowJo software. The phase ratio (\%) was calculated as the percentage of cells in the G1/S/G2 phase.

Statistical analysis. Results are expressed as the group means \pm SEM and analyzed using GraphPad Prism 5 software, using t-tests for 2-group comparisons and one-way ANOVA for three or more group comparisons. A $\mathrm{P}<0.05$ was considered to indicate a statistically significant result.

\section{Results}

miR-150 directly targets the p53 gene by interaction with the 3'-UTR. TargetScan and Pictar are two types of software broadly used on-line to predict the targets of miRNAs. Generally, the software was used to predict the targets of miRNA. In the present study, we used it to predict the target miRNA of p53. Results showed that only miR-150 targeted the 3'-UTR of p53. To confirm this, pGL3-p53-3'-UTR containing the miR-150 binding sequence and pGL3-p53-3'-mUTR were constructed (Fig. 1A and B). Analysis of the luciferase activity showed that the activity of miR-150 mimics cotransfected with pGL3-p53-3'-UTR was obviously inhibited when compared to miRNA-NC. However, the activity of miR-150 mimics cotransfected with pGL3-p53-3'-mUTR exhibited no difference when compared with miRNA-NC. Results of the luciferase activity assay indicated that mutated 3'-UTR affected the binding of miR-150 (Fig. 1C).

To further investigate whether miR-150 affects the expression of p53 at both the transcriptional and translational levels, we constructed an expression vector, pcDNA3.1-p53-3'-UTR, which contained the miR-150 binding sequence. The vector was cotransfected into H1299 cells with miR-150 mimics or
miRNA-NC. The expression level of p53 mRNA in the miR-150 mimic-transfected $\mathrm{H} 1299$ cells was significantly decreased by $47 \%$ when compared with that in the miRNA-NC-transfected cells (Fig. 1D). Moreover, the expression level of p53 protein was significantly inhibited by $60 \%$ (Fig. 1E and F).

Expression of miR-150 and its target p53 was also detected in NSCLC patient tissue samples. The clinicopathological characteristics of 13 NSCLC patients are shown in Table III. The expression of miR-150 in stage T2 tissue samples was higher than that in T1 stage tissue samples. The corresponding target gene p53 was correlated with miR-150 expression (Fig. $1 \mathrm{G}$ and $\mathrm{H}$ ). These data indicate that miR-150 directly targets p53 in NSCLC by binding to the 3 '-UTR of the p53 gene.

Overexpression of miR-150 inhibits the cell cycle arrest by targeting $p 53$. Cell cycle analysis was performed after transfection with pcDNA3.1-p53 or pcDNA3.1 for $48 \mathrm{~h}$. Results showed that the cells transfected with pcDNA3.1-p53 were significantly arrested in the G1 phase when compared to the control which was transfected with empty vector pcDNA3.1 (Fig. 2). The expression vector pcDNA3.1-p53-3'-UTR was then cotransfected into H1299 cells with the miR-150 mimics or miRNA-NC. Cell cycle analysis was also performed $48 \mathrm{~h}$ later. Both of the miR-150 mimics- or miRNA-NC-cotransfected samples exhibited an obviously cell cycle arrest in the G1 phase when compared to the control which was transfected with pcDNA3.1. However, when compared to the pcDNA3.1p53-3'-UTR- and miRNA-NC-cotransfected samples, miR-150 mimics cotransfected with pcDNA3.1-p53-3'-UTR inhibited cell cycle arrest (Fig. 3). These results indicate that miR-150 inhibits the cell cycle arrest triggered by $\mathrm{p} 53$.

Expression level of miRNAs in the H1299 cell line transfected with pcDNA3.1-p53.H1299 cell lines have a homozygous partial deletion of the p53 gene, and lack expression of p53 protein. To identify miRNAs which were differentially expressed after p53 ectopic expression in the H1299 cell line pcDNA3.1p53 was transfected into H1299 cells. To avoid targeting by miRNAs in the 3'-UTR, the pcDNA3.1-p53 contained cds only. Western blot analysis was performed to detect the expression of p53 protein in the H1299 cell line transfected with pcDNA3.1 or pcDNA3.1-p53. The data showed that the 

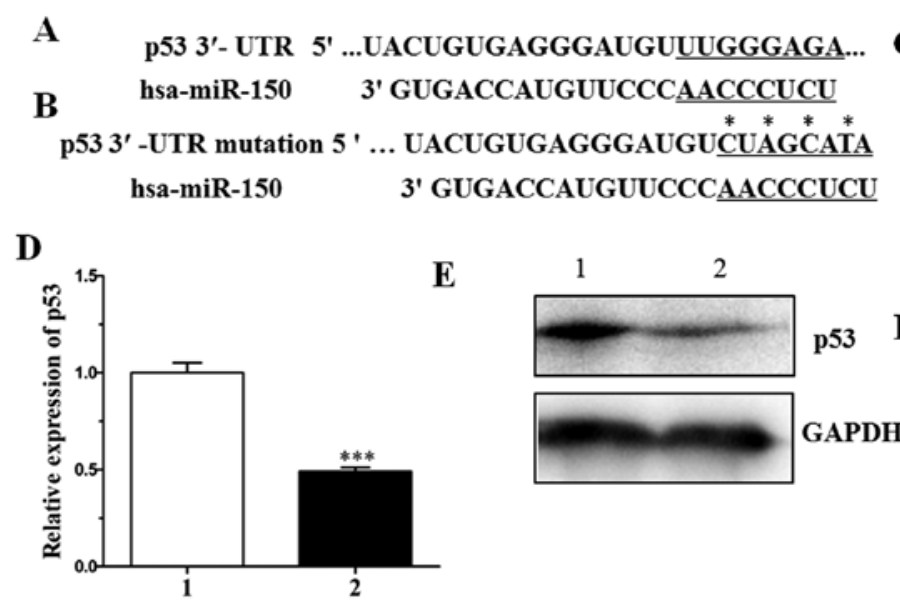

$\mathbf{E}$
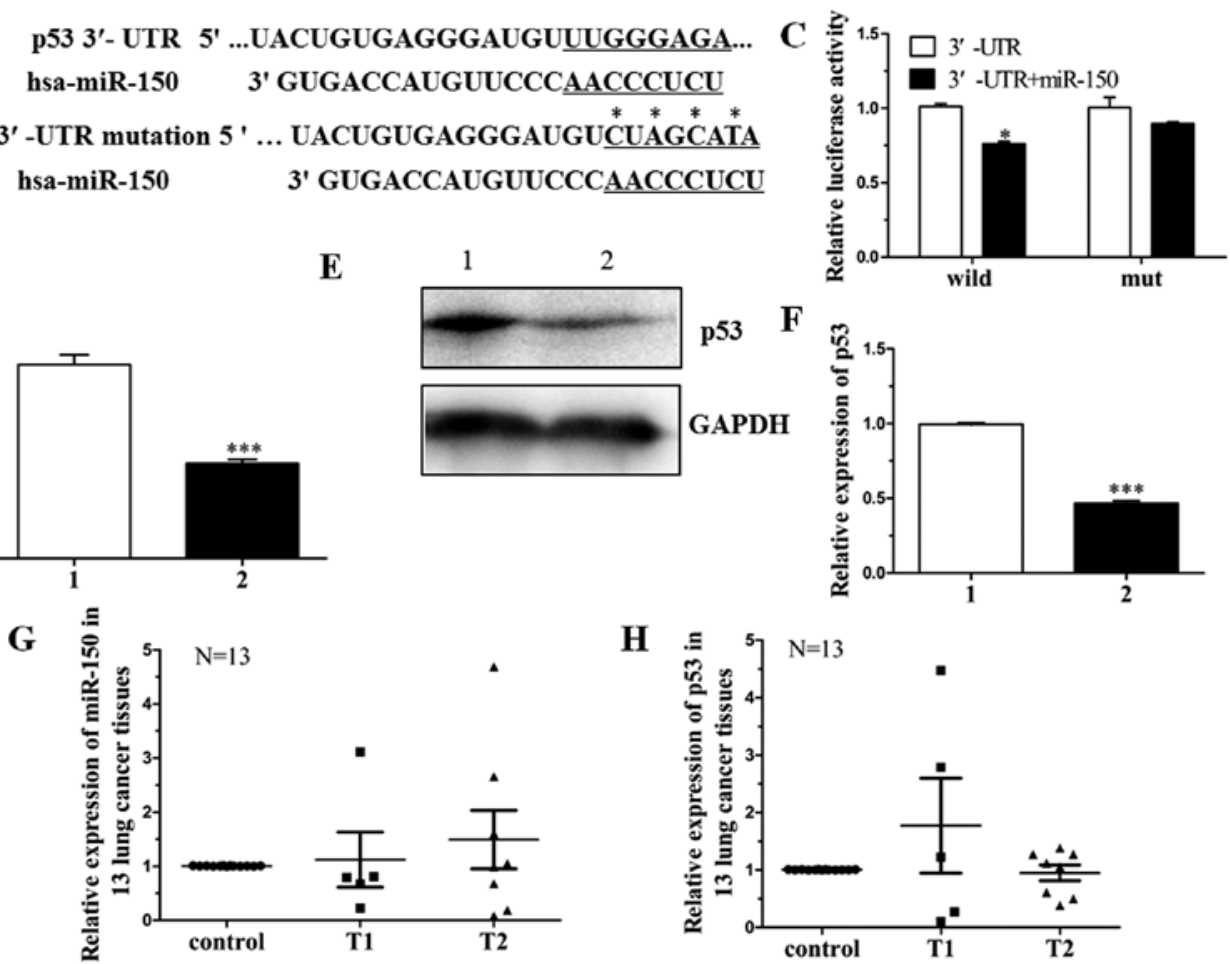

Figure 1. miR-150 directly targets the p53 gene. (A) miR-150 binding sequence in the 3'-UTR of p53. (B) Mutation of p53 3'-UTR was generated on the complementary site for the seed region of miR-150. "Indicates the mutant nucleotide. (C) Relative luciferase activity in HEK-293T cells tranfected with pGL3p53-3'-UTR or pGL3-p53-3'-mUTR and either miRNA-NC or miR-150 mimics. The normalized luciferase activity in the control group was used as the relative luciferase activity. (D-F) The expression of p53. Both samples were cotransfected with pcDNA3.1-p53-3'-UTR and either miRNA-NC or miR-150-mimics. Data in D were obtained by qRT-PCR. 18S was used as an internal control. Data in E were analyzed by western blot analysis. GAPDH was used as an internal control. Data in F showed the expression level of p53 protein analyzed by Image Lab software. ( $\mathrm{G}$ and $\mathrm{H}$ ) Expression of miR-150 and p53 was detected by qRT-PCR in 13 lung cancer patient tissues, respectively. All experiments were at least repeated in triplicate with similar results. ${ }^{*} \mathrm{P}<0.05$, ${ }^{* * *} \mathrm{P}<0.001$.

Table III. Data of the NSCLC patients.

\begin{tabular}{|c|c|c|c|c|c|c|c|}
\hline No. & Gender & $\begin{array}{l}\text { Age } \\
\text { (years) }\end{array}$ & Specimen type & Histologic type & Histology & Lymphatic invasion & pTNM \\
\hline 1 & M & 83 & Wedge resection & Adenosquamous carcinoma & GX & & T1bNOM0 \\
\hline 2 & M & 67 & Lobectomy & Adenocarcinoma & G3 & & T1bN0M0 \\
\hline 3 & M & 62 & Lobectomy & Adenosquamous carcinoma & $\mathrm{G} 2$ & & T2bNOM0 \\
\hline 4 & M & 54 & Lobectomy & Squamous cell carcinoma & G3 & & $\mathrm{T} 2 \mathrm{bN} 2 \mathrm{M} 0$ \\
\hline 5 & $\mathrm{~F}$ & 64 & Lobectomy & Adenocarcinoma & G2 & & T1bNOM0 \\
\hline 6 & $\mathrm{~F}$ & 60 & Lobectomy & Adenocarcinoma & G2 & Present & $\mathrm{T} 2 \mathrm{aN} 2 \mathrm{M} 0$ \\
\hline 7 & $\mathrm{~F}$ & 52 & Lobectomy & Adenocarcinoma (some BAC) & $\mathrm{G} 2$ & & T2bNOM0 \\
\hline 8 & M & 61 & Lobectomy & Adenocarcinoma & G1-G2 & & $\mathrm{T} 2 \mathrm{bN} 2 \mathrm{M} 0$ \\
\hline 9 & M & 62 & Lobectomy & Squamous cell carcinoma & $\mathrm{G} 2$ & Present & T2aN1M0 \\
\hline 10 & $\mathrm{~F}$ & 45 & Lobectomy & Adenocarcinoma & G2 & & T2aNOM0 \\
\hline 11 & $\mathrm{~F}$ & 67 & Lobectomy & Adenocarcinoma & $\mathrm{G} 2$ & Present & T1bN1M0 \\
\hline 12 & M & 61 & Lobectomy & Adenocarcinoma & G2 & & T2bNOM0 \\
\hline 13 & $\mathrm{~F}$ & 54 & Lobectomy & Mucoepidermoid carcinoma & G3 & Absent & T1bNOM0 \\
\hline
\end{tabular}

p53 protein was significantly expressed in the H1299 cells transfected with pcDNA3.1-p53, but was not detectable in the control (transfected with pcDNA3.1) (Fig. 4A). qRT-PCR was then performed to identify miRNAs. Results showed that the level of miR-34a, miR-184, miR-181a and miR-148 expression were significantly upregulated by 2.8-, 2.5-, 2.2- and 1.7-fold of the control (Fig. 4B). However, the expression levels of miR-10a, miR-182 and miR-34c demonstrated no difference when compared with the control. The expression values were normalized to the levels of U6 RNA. In particular, all of the 

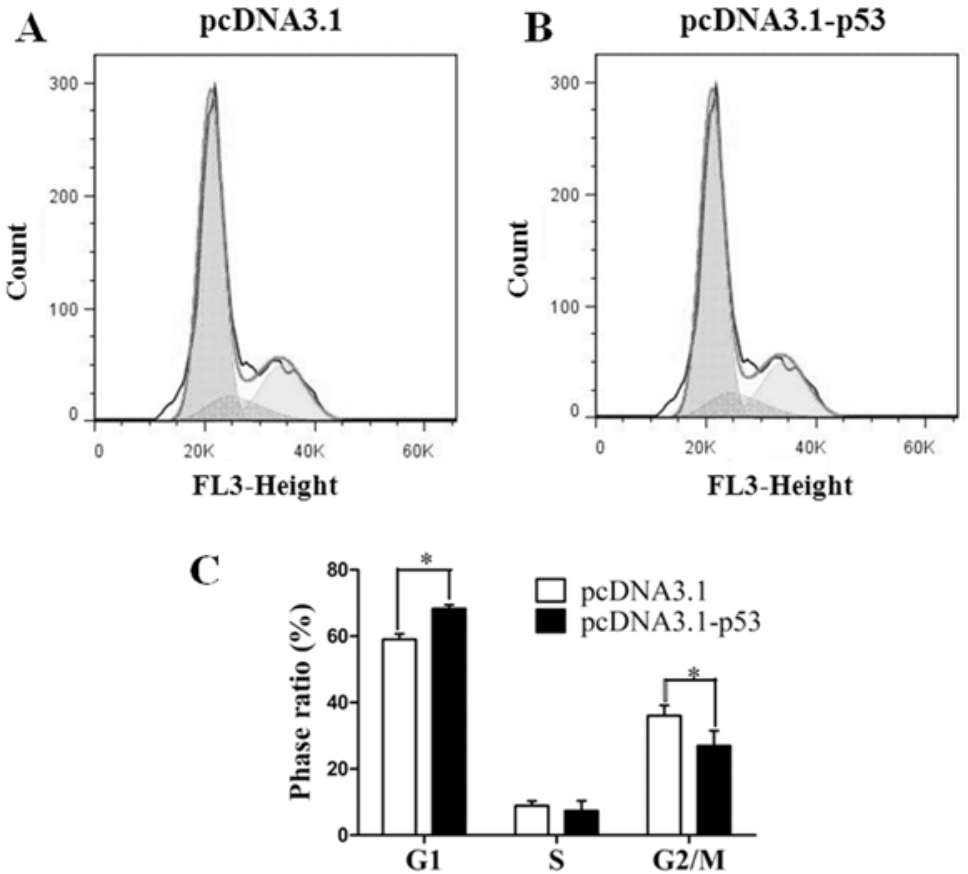

Figure 2.p53 affects the cell cycle. (A) Cell cycle distribution of H1299 cells transfected with pcDNA3.1. (B) Cell cycle distribution of H1299 cells transfected with pcDNA3.1-p53. Cell cycle distribution was measured by flow cytometry. Cell cycle analysis was performed at $48 \mathrm{~h}$ post-transfection by staining DNA with propidium iodide prior to flow cytometry. (C) The phase ratio (\%) of cells transfected with pcDNA3.1 or pcDNA3.1-p53. All experiments were at least repeated in triplicate with similar results. ${ }^{*} \mathrm{P}<0.05$.

A
pcDNA3.1

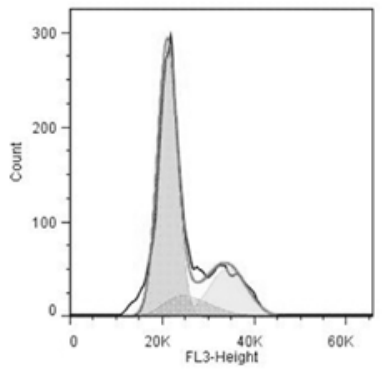

C pcDNA3.1-p53-3'-UTR

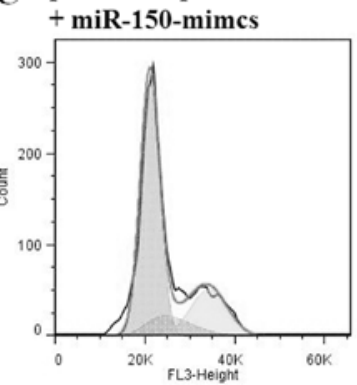

B pcDNA3.1-p53-3'-UTR + miRNA-NC

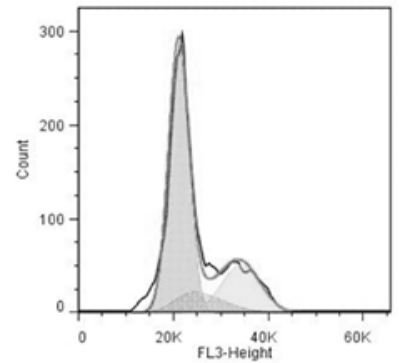

D

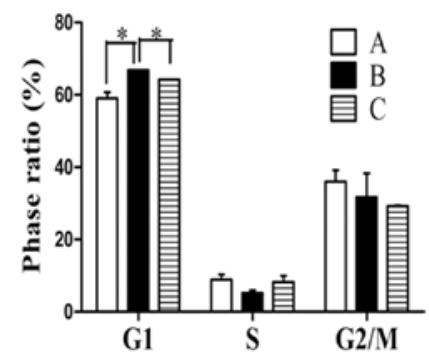

Figure 3. miR-150 affects the cell cycle distribution by targeting p53. (A-C) Cell cycle distribution measured by flow cytometry. Cell cycle analysis was performed at $48 \mathrm{~h}$ post-transfection by staining DNA with propidium iodide prior to flow cytometry. (D) The phase ratio (\%) of cells transfected with pcDNA3.1 or pcDNA3.1-p53-3'-UTR with either miRNA-NC or miR-150-mimcs. All experiments were at least repeated in triplicate with similar results. ${ }^{*} \mathrm{P}<0.05$.

upregulated miRNAs play a cancer-suppressor role in lung cancer tumorigenesis which has been previously reported (21-27). Thus, these data indicate that p53 protein promotes

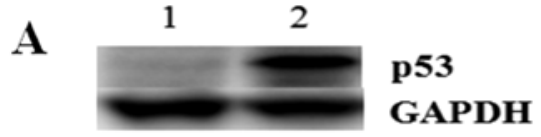

$\mathbf{B}$

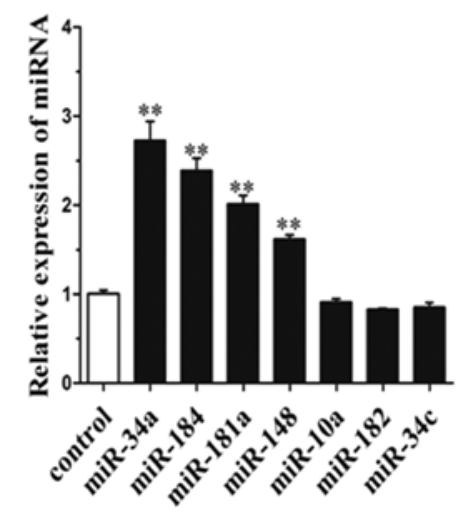

Figure 4. Expression of p53 protein and miRNAs in H1299 cells transfected with pcDNA3.1-p53. (A) The expression level of p53 protein. Lanes 1 and 2 indicate control (transfected with pcDNA3.1) and pcDNA3.1-p53, respectively. (B) Expression of miRNAs. Data in A were obtained by western blot analysis. GAPDH was used as an internal control. Data in B were obtained by qRT-PCR. U6 was used as an internal control. Each assay was performed in triplicate. ${ }^{* *} \mathrm{P}<0.01$.

the expression of miRNAs, particularly tumor suppressors miR-34a and miR-184.

Expression level of miR-34a, miR-184 and $p 53$ was relevant in NSCLC cell lines. To confirm that the expression level of 
A

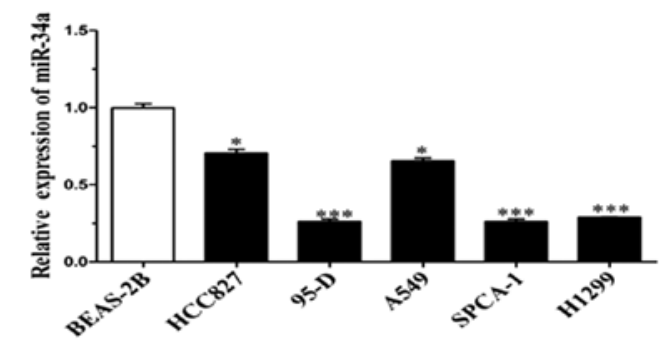

B

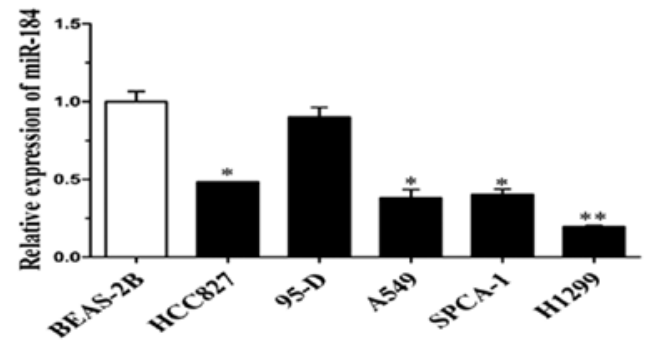

C

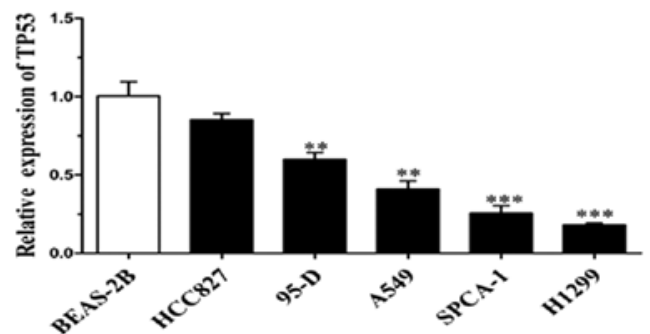

Figure 5. Expression of miR-34a, miR-184 and p53 in lung cancer cell lines. Expression of (A) miR-34a, (B) miR-184 and (C) p53. Data were performed by qRT-PCR. The expression level of each gene is the fold-change relative to the expression level of BEAS-2B. U6 and 18S were used as the interna control in A, B and C respectively. Each assay was performed in triplicate. ${ }^{*} \mathrm{P}<0.05,{ }^{* *} \mathrm{P}<0.01,{ }^{* * *} \mathrm{P}<0.001$.

miR-34a, miR-184 and p53 was relevant, 5 lung cancer cell lines (A549, H1299, 95-D, SPCA-1 and HCC827) were chosen as the samples and normal lung cell line (BEAS-2B) as the control. qRT-PCR analysis was performed to detect the expression levels of miR-34a, miR-184 and p53. The data showed that the expression of miR-34a and miR-184 was consistent with p53 except for that in the 95-D cell line (Fig. 5). Notably, all of the other 4 lung cancer cell lines originated from NSCLC. Altogether, these results indicate that $\mathrm{p} 53$ protein affects the expression of miR-34a and miR-184.

miR-150, $p 53$ protein and miRNAs are members of a regulatory network in NSCLC tumorigenesis. As the results confirmed, miR-150 targets the 3'-UTR of p53. Overexpression of p53 can significantly enhance the expression of miR-34a, miR-184, miR-181a and miR-148. In particular, the expression of miR-34a and miR-184 was increased higher than 2-fold of the control. The targets of miR-34a have been previously reported $(21,28,29)$. The protein cyclin E2 (CCNE2) is a key regulator in the cell cycle, and it is a potential target of miR-34a (Table I). miR-181a and miR-148 regulate the expression of CDC73 and CDK1, respectively. Both CDC73 and CDK1 can affect the G1 phase in the cell cycle (24,30-36). Thus, miR-150, p53 protein, the relevant miRNAs and their targets may consist of a complicated regulation network in NSCLC tumorigenesis (Fig. 6).

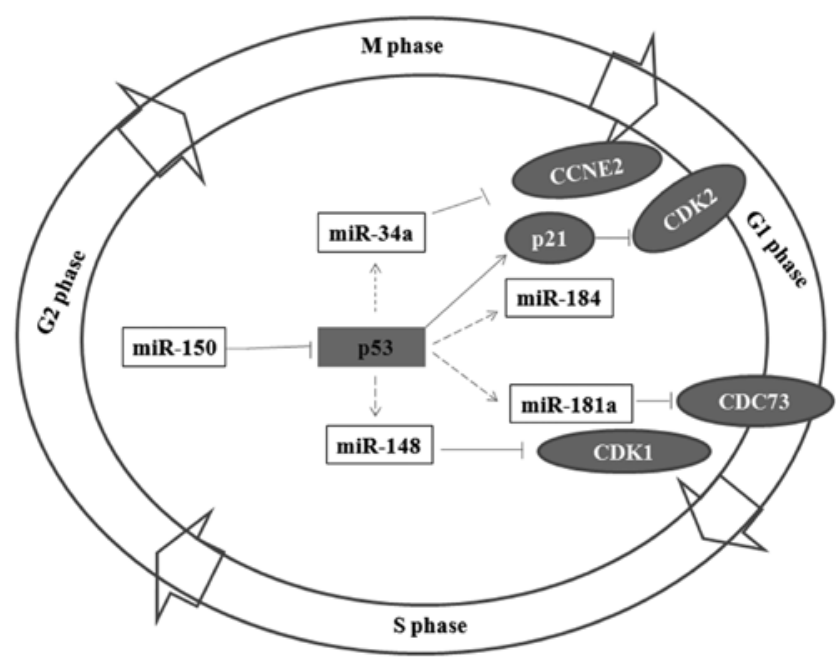

Figure 6. Role of miR-150, p53 and relevant miRNAs in the regulatory network among miRNA and protein factors. miR-150 inhibits the expression of $\mathrm{p} 53$, however, $\mathrm{p} 53$ promotes the expression of miR-34a, miR-184, miR-148 and miR-181a.

\section{Discussion}

H1299 cells have a homozygous partial deletion of the p53 gene, and lack expression of p53 protein (37). In the present study, we detected the expression variation of miRNAs when ectopic expression of $\mathrm{p} 53$ was present in the $\mathrm{H} 1299$ cell line. We found that miR-34a, miR-184, miR-181a and miR-148 expression was significantly upregulated. miR-34a and miR-181a have been reported as tumor-suppressor genes in neuroblastoma cells, urothelial bladder carcinoma, human brain glioma cells, head and neck squamous cell carcinoma and breast cancer (21-23,38-40). miR-34a can target many protein factors, such as the Notch-1 signaling pathway, Bcl-2, SIRT-1 and CDK1 and then promote the process of cell apoptosis and inhibit the cell cycle and proliferation $(22,23,41,42)$. miR-181a can target k-ras, a typical oncogene (39). However, miR-184 and miR-148 have not been thoroughly studied. These results suggest that p53 protein may regulate the expression of various miRNAs which play a tumor-suppressor role in NSCLC cell lines. Yet, how $\mathrm{p} 53$ protein affects the expression of miRNAs is still unknown.

miR-150 was the only predicted miRNA which binds to the 3'-UTR sequence of p53. The luciferase activity analysis showed that the activity of miR-150 mimics cotransfected with pGL3-p53-3'-UTR was inhibited obviously compared to miRNA-NC. Western blot analysis also showed consistent results that the translation of $\mathrm{p} 53$ protein was inhibited significantly when miR-150 mimics were cotransfected with pcDNA3.1-p53-3'-UTR. miR-150 also reduces the cell cycle arrest triggered by p53. These results suggest that miR-150 may promote lung cancer tumorigenesis by targeting p53.

In conclusion, we confirmed that p53 is a direct target of miR-150, and overexpression of p53 promotes the expression of miRNAs including miR-34a, miR-184, miR-181a and miR-148. Our findings suggest that miR-150, p53 protein and relevant miRNAs consist of a complicated regulatory network in NSCLC tumorigenesis. 


\section{Acknowledgements}

This study was in part supported by grants from the Innovation Program of Shanghai Municipal Commission of Sciences and Technology (11ZR141220), the National Natural Science Foundation (31170750), the National Key Research and Development Program of China (2011CB811304), the National Basic Research Program of China (2011CBA01105) and the Program of Baoshan District Commission of Sciences and Technology, Shanghai (CXY-2011-32).

\section{References}

1. Zabaleta J: MicroRNA: a bridge from H. pylori infection to gastritis and gastric cancer development. Front Genet 3: 294, 2012.

2. Harquail J, Benzina S and Robichaud GA: MicroRNAs and breast cancer malignancy: an overview of miRNA-regulated cancer processes leading to metastasis. Cancer Biomark 11: 269-280, 2012

3. Wang F, Sun GP, Zou YF, Hao JQ, Zhong F and Ren WJ: MicroRNAs as promising biomarkers for gastric cancer. Cancer Biomark 11: 259-267, 2012.

4. Odjele A, Charest D and Morin PJ: miRNAs as important drivers of glioblastomas: a no-brainer? Cancer Biomark 11: 245-252, 2012.

5. Eldem V, Celikkol Akcay U, Ozhuner E, Bakir Y, Uranbey S and Unver T: Genome-wide identification of miRNAs responsive to drought in peach (Prunus persica) by high-throughput deep sequencing. PloS One 7: e50298, 2012.

6. Endo H, Muramatsu T, Furuta M, et al: Potential of tumorsuppressive miR-596 targeting LGALS3BP as a therapeutic agent in oral cancer. Carcinogenesis 34: 560-569, 2013.

7. Li KK, Pang JC, Lau KM, et al: miR-383 is downregulated in medulloblastoma and targets peroxiredoxin 3 (PRDX3). Brain Pathol: Dec 11, 2012 (Epub ahead of print). doi: 10.1111/ bpa.12014

8. Masuda M, Miki Y, Hata S, et al: An induction of microRNA, miR-7 through estrogen treatment in breast carcinoma. J Trans Med 10 (Suppl 1): S2, 2012.

9. Novello C, Pazzaglia L, Cingolani C, et al: miRNA expression profile in human osteosarcoma: role of miR-1 and miR-133b in proliferation and cell cycle control. Int J Oncol 42: 667-675, 2013.

10. Ando H, Okamoto A, Yokota M, et al: Development of miR-92a delivery system for antiangiogenesis-based cancer therapy. J Gene Med 15: 20-27, 2012.

11. Teixeira AL, Gomes M and Medeiros R: EGFR signaling pathway and related-miRNAs in age-related diseases: the example of miR-221 and miR-222. Front Genet 3: 286, 2012.

12. Boldrup L, Coates PJ, Wahlgren M, Laurell G and Nylander K Subsite-based alterations in miR-21, miR-125b, and miR-203 in squamous cell carcinoma of the oral cavity and correlation to important target proteins. J Carcinog 11: 18, 2012.

13. Hirata H, Ueno K, Shahryari V, et al: Oncogenic miRNA-182-5p targets Smad4 and RECK in human bladder cancer. PloS One 7: e51056, 2012.

14. Hassan F, Nuovo GJ, Crawford M, et al: MiR-101 and miR-144 regulate the expression of the CFTR chloride channel in the lung. PloS One 7: e50837, 2012.

15. Sasaki A, Udaka Y, Tsunoda Y, et al: Analysis of p53 and miRNA expression after irradiation of glioblastoma cell lines. Anticancer Res 32: 4709-4713, 2012.

16. Suzuki HI and Miyazono K: p53 actions on microRNA expression and maturation pathway. Methods Mol Biol 962: 165-181, 2013.

17. Gurtler A, Kunz N, Gomolka M, et al: Stain-Free technology as a normalization tool in Western blot analysis. Anal Biochem 433 105-111, 2012.

18. Hirano S: Western blot analysis. Methods Mol Biol 926: 87-97, 2012.

19. Kao $\mathrm{CH}$, Cheng $\mathrm{CM}$, Chuang $\mathrm{KH}$, et al: A regularly spaced and self-revealing protein ladder for anti-tag Western blot analysis. Anal Biochem 431: 1-3, 2012

20. Nybo K: Molecular biology techniques Q\&A. Western blot: protein migration. Biotechniques 53: 23-24, 2012.
21. Li XJ, Ji MH, Zhong SL, et al: MicroRNA-34a modulates chemosensitivity of breast cancer cells to adriamycin by targeting Notch1. Arch Med Res 43: 514-521, 2012.

22. Siemens H, Neumann J, Jackstadt R, et al: Detection of miR-34a promoter methylation in combination with elevated expression of c-Met and $\beta$-catenin predicts distant metastasis of colon cancer. Clin Cancer Res 9: 710-720, 2012.

23. Zhang HS, Chen XY, Wu TC, Sang WW and Ruan Z: MiR-34a is involved in Tat-induced HIV-1 long terminal repeat (LTR) transactivation through the SIRT1/NFkappaB pathway. FEBS Lett 586: 4203-4207, 2012.

24. Candas D, Fan M, Nantajit D, et al: CyclinB1/Cdk1 phosphorylates mitochondrial antioxidant MnSOD in cell adaptive response to radiation stress. J Mol Cell Biol: Jan 31, 2013 (Epub ahead of print).

25. Haflidadottir BS, Bergsteinsdottir K, Praetorius C and Steingrimsson E: miR-148 regulates Mitf in melanoma cells. PloS One 5: e11574, 2010

26. Iovino N, Pane A and Gaul U: miR-184 has multiple roles in Drosophila female germline development. Dev Cell 17: 123-133, 2009.

27. Liu C, Teng ZQ, Santistevan NJ, et al: Epigenetic regulation of miR-184 by MBD1 governs neural stem cell proliferation and differentiation. Cell Stem Cell 6: 433-444, 2010.

28. Chen Y, Tsai YH and Tseng SH: Inhibition of cyclin-dependent kinase 1-induced cell death in neuroblastoma cells through the microRNA-34a-MYCN-survivin pathway. Surgery 153: 4-16, 2013.

29. Zhang C, Yao Z, Zhu M, et al: Inhibitory effects of microRNA34a on cell migration and invasion of invasive urothelial bladder carcinoma by targeting Notch1. J Huazhong Univ Sci Technolog Med Sci 32: 375-382, 2012.

30. Venta R, Valk E, Koivomagi M and Loog M: Double-negative feedback between S-phase cyclin-CDK and CKI generates abruptness in the G1/S switch. Front Physiol 3: 459, 2012.

31. Nagle AA, Gan FF, Jones G, So CL, Wells G and Chew EH: Induction of tumor cell death through targeting tubulin and evoking dysregulation of cell cycle regulatory proteins by multifunctional cinnamaldehydes. PloS One 7: e50125, 2012.

32. Chow JP and Poon RY: The CDK1 inhibitory kinase MYT1 in DNA damage checkpoint recovery. Oncogene: Nov 12, 2012 (Epub ahead of print).

33. Guarnieri V, Battista C, Muscarella LA, et al: CDC73 mutations and parafibromin immunohistochemistry in parathyroid tumors: clinical correlations in a single-centre patient cohort. Cell Oncol (Dordr) 35: 411-422, 2012.

34. Amrich CG, Davis CP, Rogal WP, et al: Cdc73 subunit of Paf1 complex contains C-terminal Ras-like domain that promotes association of Paf1 complex with chromatin. J Biol Chem 287: 10863-10875, 2012.

35. Zhang JH, Seigneur EM, Pandey M, et al: The EIF4EBP3 translational repressor is a marker of CDC73 tumor suppressor haploinsufficiency in a parathyroid cancer syndrome. Cell Death Dis 3: 266, 2012

36. Masi G, Barzon L, Iacobone M, et al: Clinical, genetic, and histopathologic investigation of CDC73-related familial hyperparathyroidism. Endocr Relat Cancer 15: 1115-1126, 2008.

37. Jiang Y, Zhang XY, Sun L, et al: Methyl methanesulfonate induces apoptosis in p53-deficient H1299 and Hep3B cells through a caspase 2- and mitochondria-associated pathway. Environ Toxicol Pharmacol 34: 694-704, 2012.

38. Debernardi S, Skoulakis S, Molloy G, Chaplin T, DixonMcIver A and Young BD: MicroRNA miR-181a correlates with morphological sub-class of acute myeloid leukaemia and the expression of its target genes in global genome-wide analysis. Leukemia 21: 912-916, 2007.

39. Shin KH, Bae SD, Hong HS, Kim RH, Kang MK and Park NH: miR-181a shows tumor suppressive effect against oral squamous cell carcinoma cells by downregulating K-ras. Biochem Biophys Res Commun 404: 896-902, 2011.

40. Xie W, Li Z, Li M, Xu N and Zhang Y: miR-181a and inflammation: miRNA homeostasis response to inflammatory stimuli in vivo. Biochem Biophys Res Commun 430: 647-652, 2012.

41. Kashat M, Azzouz L, Sarkar SH, Kong D, Li Y and Sarkar FH: Inactivation of AR and Notch-1 signaling by miR-34a attenuates prostate cancer aggressiveness. Am J Transl Res 4: 432-442, 2012.

42. Xia J, Duan Q, Ahmad A, et al: Genistein inhibits cell growth and induces apoptosis through up-regulation of miR-34a in pancreatic cancer cells. Curr Drug Targets 13: 1750-1756, 2012. 